\title{
A Positioning Method to Geostationary Satellite by TWSTFT Links
}

\author{
Hongwei Sun, Weiqing Yang and Yuli Li \\ Xi'an University, Kejiliulu 1,710065, Xi’an, Shaanxi, China
}

\begin{abstract}
Based on the Asia-Pacific two-way satellite time and frequency transfer (TWSTFT) links, an accurate positioning method to geostationary satellite is presented, and the accuracy of the positioning is also estimated. The characteristics of the method are simple, practical and accurate. Since the system is established on an existent system that is for the international time transfer, it dose not need a big investment. At last, some future applications via the accurate satellite position are mentioned.
\end{abstract}

Keywords—TWSTFT; geostationary satellite; positioning

\section{INTRODUCTION}

TWSTFT technique is at present one of the most precise and accurate time comparison method, and the Bureau International des Poids et des Mesures (BIPM) has already adopted the TWSTFT data for the computation of the International Atomic Time (TAI) and Coordinated Universal Time (UTC). In Asia-Pacific region, a TWSTFT links including some important time laboratories has been constructed. In the system, geostationary communication satellite is used as their relay stations. Moreover, in some links of time comparisons a common geostationary communication satellite is used. In addition, we have been developing a new time transfer modem, it can realize simultaneous time transfer among ground stations up to 8 stations. From the two aspects above, we present an application of the Asia-Pacific TWSTFT, that is, an accurate positioning method to the geostationary communication satellite by Asia-Pacific TWSTFT network. About the positioning to satellite, there are several methods, it mainly includes the ranging methods by radio signal. For example, for the geostationary meteorological satellite (GMS), its position is determined by the ranging from 3 stations, that is, in main station the radio signals (S-band) are emitted to the satellite, then the main station and 2 slave stations receive the signals from the satellite. Once the 2 slave stations receive the signal, the signal is reemitted to the satellite, and the main station receive the signals as well as calculate the position of the satellite by three delay data. The method is practical and reliable, but the positioning accuracy is limited by differential propagation delay and poor geometric distribution among stations and the satellite. Another method of positioning to satellite is laser ranging that possesses a more accuracy, but its measurements depend strongly on the weather conditions, and an establishment of the system needs a big investment. With the science and technology progress, there are more and more research fields, such as, high precise time dissemination or navigation via satellites, need to know the accurate position of the satellite. So the research of determining position of satellite is significant.

\section{Method OF The Positioning}

The Asia-Pacific TWSTFT network includes main time laboratories in the region. They are CRL (Communications Research Laboratory, Japan); NRLM (National Research Laboratory of Metrology, Japan); TL (Telecommunication Laboratories, Chinese Taipei); NTSC (Ntional Time Serve Center, China); KRISS (Korea Research Institute of Standards and Science, Korea); PSB (Productivity and Standards Board, Singapore). These laboratories use a common geostationary communication satellite located $150^{\circ} \mathrm{E}$ for the TWSTFT experiments.

We choose at least 4 stations from them in order to determine the satellite position. As an example, if there is 4 stations (that is, CRL; NRLM; TL; NTSC) chosen, when carrying out simultaneously time transfer among them, we can get 3 time delay data from NRLM to CRL; TL to CRL; NTSC to CRL.

Suppose the 4 ground stations are marked by G1; G2; G3; G4, their coordinates are marked by (xi, yi, zi), i = 1, 2, 3, 4 . G4 (CRL), as the main station where receives and processes all delay data, is charge of the positioning system.

In order to illustrate the principle of the positioning simply, we assume the no path delay error in $\mathrm{Ri}$ which is the delay from Gi to G4 via the satellite is eliminated, then the positioning equations can be obtained as follows.

$$
\begin{aligned}
& \operatorname{Ri} \times C= \sqrt{\left(x_{i}-x_{s}\right)^{2}+\left(y_{i}-y_{s}\right)^{2}+\left(z_{i}-z_{s}\right)^{2}} \\
&+\sqrt{\left(x_{4}-x_{s}\right)^{2}+\left(y_{4}-y_{s}\right)^{2}+\left(z_{4}-z_{s}\right)^{2}} \\
& \mathrm{i}=1,2,3
\end{aligned}
$$

where (xs, ys, xs) is the satellite coordinates, C is light speed.

Since the coordinates of the stations and the delays are known, and equation group (1) includes 3 unknown numbers xs, ys, zs, we can calculate out the satellite position.

If we use two-way delay data in TWSTFT, such as, from TL to CRL and CRL to TL, or use more data from more ground stations, the number of equation in (1) will increase, which will improve the precision of positioning. In order to increase the position precision and the system reliability, all 
stations in the TWSTFT network and their two-way delay data should attend the positioning calculation.

\section{A. Positioning Accuracy}

As usual the positioning accuracy for satellites depends on two aspects. One is the geometric distribution among stations and the satellite, another is the ranging accuracy, next we analysis the problems in detail.

Firstly, we discuss the effect of the geometric distribution to the positioning accuracy. For the equation group (1), by linear developing method to delete its high order terms, we can obtain:

$$
\left[\begin{array}{lll}
A 11 & A 12 & A 13 \\
A 21 & A 22 & A 23 \\
A 31 & A 32 & A 33
\end{array}\right]\left[\begin{array}{l}
\Delta x \\
\Delta y \\
\Delta z
\end{array}\right]=\left[\begin{array}{l}
b 1 \\
b 2 \\
b 3
\end{array}\right]
$$

where

$$
\begin{array}{cc}
\text { Ai1 }=(\mathrm{xi}-\mathrm{x} 0) / \mathrm{di}+(\mathrm{x} 4-\mathrm{x} 0) / \mathrm{d} 4 & \mathrm{i}=1,2,3 \\
\mathrm{Ai} 2=(\mathrm{yi}-\mathrm{y} 0) / \mathrm{di}+(\mathrm{y} 4-\mathrm{y} 0) / \mathrm{d} 4 & \mathrm{i}=1,2,3 \\
\mathrm{Ai} 3=(\mathrm{zi}-\mathrm{z} 0) / \mathrm{di}+(\mathrm{z} 4-\mathrm{z} 0) / \mathrm{d} 4 & \mathrm{i}=1,2,3 \\
\mathrm{di}=\sqrt{\left(x_{i}-x_{s}\right)^{2}+\left(y_{i}-y_{s}\right)^{2}+\left(z_{i}-\mathrm{zs}_{s}\right)^{2}} & \mathrm{i}=1,2,3,4 \\
\mathrm{bi}=\mathrm{Ri} \times \mathrm{C}-(\mathrm{di}+\mathrm{d} 4) & \mathrm{i}=1,2,3 \\
\triangle \mathrm{x}=\mathrm{xs}-\mathrm{x} 0 ; \triangle \mathrm{y}=\mathrm{ys}-\mathrm{y} 0 ; \triangle \mathrm{z}=\mathrm{zs}-\mathrm{z} 0
\end{array}
$$

$(\mathrm{x} 0, \mathrm{y} 0, \mathrm{z} 0)$ is the normal position of the satellite.

The equation group (2) can be indicated as

$$
\mathrm{AX}=\mathrm{B}
$$

where $A=(A i j), i, j=1,2,3$, it is called a coefficient matrix.

$$
\begin{gathered}
\mathrm{X}=(\triangle \mathrm{x}, \triangle \mathrm{y}, \triangle \mathrm{z}), \\
\mathrm{B}=(\mathrm{b} 1, \mathrm{~b} 2, \mathrm{~b} 3) .
\end{gathered}
$$

The lowest quadratic solution of equation (3) is

$$
\mathrm{X}=\left(\mathrm{A}^{T} \mathrm{~A}\right)^{-1} \mathrm{~A}^{T} \mathrm{~B}
$$

where $\mathrm{A}^{T}=($ Aji) $\mathrm{j}, \mathrm{i}=1,2,3$.

Thus, the coordinate variance matrix of $\mathrm{X}$ is

$$
\operatorname{Cov}(\mathrm{X})=\left(\mathrm{A}^{T} \mathrm{~A}\right)^{-1} \mathrm{~A}^{T} \mathrm{~B} \operatorname{Cov}(\mathrm{B})\left[\left(\mathrm{A}^{T} \mathrm{~A}\right)^{-1} \mathrm{~A}^{T} \mathrm{~B}\right]^{T}(5)
$$

When the geometric distribution of ground stations is only considered, we assume the $\operatorname{Cov}(\mathrm{B})$ is unit matrix, then

$$
\operatorname{Cov}(\mathrm{X})=\left(\mathrm{A}^{T} \mathrm{~A}\right)^{-1}=\left[\begin{array}{ccc}
\sigma_{x x}^{2} & \sigma_{x y}^{2} & \sigma_{x z}^{2} \\
\sigma_{y x}^{2} & \sigma_{y y}^{2} & \sigma_{y z}^{2} \\
\sigma_{z x}^{2} & \sigma_{z y}^{2} & \sigma_{z z}^{2}
\end{array}\right]
$$

The square root of the sum of elements in diagonal line of the matrix is defined usually as a judgment standard of the geometric distribution among ground stations and the satellite, the value is called position dilution of precision (PDOP).

$$
\mathrm{PDOP}=\sqrt{\sigma_{x x}^{2}+\sigma_{y y}^{2}+\sigma_{z z}^{2}}
$$

According to the definition accepted extensively, the positioning error equal the ranging error multiplied by PDOP. Thus the less the PDOP is, the less the positioning error. Generally speaking, the larger the area surrounded by these ground stations is, the less the PDOP is. So we should choose the stations with a big latitude and longitude difference each other, in other word, the stations should be distributed in wider region. In addition, we have proved that by adding stations the PDOP will be decreased. Table 1 gives some values of PDOP among the stations in the TWSTFT network, the station' number of attending the positioning calculation is from 4 to 6 .

TABLE I. SOME PDOP VALUES

\begin{tabular}{|c|c|c|c|c|c|c|}
\hline CRL & NRLM & PSB & TL & NTSC & KRISS & PDOP \\
\hline $\mathrm{Y}$ & $\mathrm{Y}$ & $\mathrm{Y}$ & $\mathrm{Y}$ & $\mathrm{Y}$ & $\mathrm{Y}$ & 30.9 \\
\hline $\mathrm{Y}$ & $\mathrm{Y}$ & $\mathrm{Y}$ & & $\mathrm{Y}$ & $\mathrm{Y}$ & 31.0 \\
\hline $\mathrm{Y}$ & $\mathrm{Y}$ & $\mathrm{Y}$ & $\mathrm{Y}$ & & $\mathrm{Y}$ & 54.4 \\
\hline $\mathrm{Y}$ & $\mathrm{Y}$ & $\mathrm{Y}$ & $\mathrm{Y}$ & $\mathrm{Y}$ & & 31.3 \\
\hline $\mathrm{Y}$ & $\mathrm{Y}$ & $\mathrm{Y}$ & & $\mathrm{Y}$ & & 31.4 \\
\hline $\mathrm{Y}$ & $\mathrm{Y}$ & $\mathrm{Y}$ & $\mathrm{Y}$ & & $\mathrm{Y}$ & 77.8 \\
\hline
\end{tabular}

\section{B. Ranging Accuracy}

There are 4 aspects that affect the ranging accuracy. We give an estimate concisely as follows.

\section{1) Delay error from the propagation path}

The signal path from one station to other station via the geostationary communication satellite is mainly free space with a small amount of ionosphere and troposphere. When the signal through the ionosphere and troposphere, the delay will increase. For Ku-band frequency in the TWSTFT, the delay correction amount from ionosphere is very small. It is calculated by following formula.

$$
\triangle \mathrm{T}=134.2 \times \mathrm{Ne} / \mathrm{f}^{2}
$$

where $\mathrm{f}$ is the signal frequency; Ne is TEC.

According to our analysis, when the elevation from station 
to satellite is bigger than $30^{\circ}$, the corrective error will be less than 0.5 ns.

The delay from the troposphere is related to the elevation at stations. The table 2 gives the elevations at some stations. As usual the error of delay correction amount may be less than $1 \mathrm{~ns}$.

TABLE II. ELEVATIONS FROM STATIONS TO SATELLITE

\begin{tabular}{|l|l|l|c|}
\hline station & elevation & station & elevation \\
\hline & unit: degree & & unit: degree \\
\hline CRL & 47.2 & KRISS & 41 \\
\hline NRLM & 47 & PSB & 37.1 \\
\hline TL & 46.7 & NTSC & 31.3 \\
\hline
\end{tabular}

\section{2) Delay error from the equipment at ground stations}

For the equipment that we have developed recently, their delay may be calibrated automatically, and the uncertainty is less than 2 ns.

\section{3) The error of time synchronization among the stations}

Because the time among the stations can be synchronized by the TWSTFT itself, and the accuracy is less than 2 ns.

\section{4) Coordinate error in stations}

By the GPS or other means, it is easy to determine the station' position with error of $1 \mathrm{~m}$ (correspond to $3.3 \mathrm{~ns}$ ).

From these mentioned above, we can obtain the ranging accuracy (RA).

$$
\mathrm{RA}=\sqrt{0.5^{2}+1^{2}+2^{2}+2^{2}+3.3^{2}} \approx 4.5(\mathrm{~ns})
$$

When CRL; NRLM; PSB; CSAO attend the positioning calculation, the positioning accuracy (PA) is

$$
\mathrm{PA}=\mathrm{PDOP} \times \mathrm{RA}=31.4 \times 4.5 \approx 141(\mathrm{~ns}) \approx 42(\mathrm{~m})(10)
$$

\section{SYSTEM CHARACTERISTICS AND CONCLUSIONS}

By the Asia-Pacific TWSTFT links to determine the geostationary communication satellite position is a new method, it processes several advantages.

The positioning system has a high reliability, since the laboratories in the network are all the most important time and frequency research institutes with keeping the high precise time scales in their county, moreover, they have important position in the international organization (BIPM). They are able to keep a consecutive longterm operations.

In order to get an accurate positioning, the time of the stations must be synchronized with a high accuracy. The TWSTFT itself just possesses such an accurate time transfer ability among these stations.
With the high frequency (Ku-band) in the TWSTFT, the ionospheric effect to the ranging error is smaller.

These elevations from the stations to the satellite in these laboratories are relative big, so the ionospheric and tropospheric effects to ranging error are smaller.

There is a good geometric distribution among the stations, so the PDOP is smaller.

The positioning system is established on existent system, and the positioning method is simple and easy to carry on, so it does not need a big investment.

The system provides an important condition for other application researches, such as, an establishment of satellite time service or satellite navigation system that depends on mainly the accurate positioning to the satellite.

From the analyses above, it is seen the positioning method from the TWSTFT network being practical and accurate. If such a system can be accomplished, it will open a new way of many application researches.

\section{ACKNOWLEDGMENT}

This work is supported by Science and Technology Plan Projects of Xian (No. CXY1352WL27) and Natural Science Basic Research Plan in Shaanxi Province (No. 2017JM6100), and the authors thank the organizations.

\section{REFERENCES}

[1] Barnes, A.: 'Characterization of frequency stability’, IEEE Transactions on Instrumentation and Measurement, 21, pp. 11-20, 1997.

[2] Schmidt, S.: 'Atomic clock models using fractionally integrated noise processes', Metrologia, 40, pp. 305-311, 2003.

[3] Allan, W.: 'Time and frequency (time-domain) characterization, estimation, and prediction of precision clocks and oscillators', IEEE Transactions on Ultrasonics, Ferroelectrics, and Frequency Control, 34, pp. 647-654, 1987. 